\title{
Generation of Entanglement between Two Two-Level Atoms Coupled to a Microtoroidal Cavity Via Thermal Field
}

\author{
Emilio H. S. Sousa * (D) and J. A. Roversi ${ }^{(D)}$ \\ Instituto de Fisica "Gleb Wataghin”, Universidade Estadual de Campinas, Campinas 13083970, SP, Brazil; \\ roversi@ifi.unicamp.br \\ * Correspondence: ehssousa@ifi.unicamp.br
}

Received: 15 June 2020; Accepted: 7 July 2020; Published: 8 July 2020

check for updates

\begin{abstract}
We investigate the entanglement dynamics of a system comprising a pair of two-level dipole-dipole interacting atoms coupled to a microtoroidal resonator. Each atom is individually coupled with the two counter-propagating whispering gallery modes of the resonator through their evanescent fields. The atom-atom entanglement shown for several parameter sets of the system was obtained using the negativity. For ideal resonators, it is seen that the entanglement is correlated to the dipole-dipole interaction and the average number of photons when the modes of the resonator are prepared in a thermal state even at high temperatures. Further, for the non-ideal resonator case, where there is a small structural deformation of the microtoroidal structure that allows a direct coupling between the modes, a counter-intuitive result is presented. The imperfections also offer the advantage of generating maximally entangled states for a two-atom subsystem with maximum fidelity.
\end{abstract}

Keywords: entanglement; microtoroidal resonator; two two-level atoms; thermal field

\section{Introduction}

Quantum entanglement represents a key resource for exploring quantum information processing, such as quantum cryptography [1], quantum computing [2], and quantum teleportation [3]. In this context, the cavity quantum electrodynamics (cavity QED) has emerged as one of the most promising candidates for the creation, manipulation, and exploitation of entanglement via controlled interactions [4,5]. Such cavity QED systems have been extensively studied, both experimentally and theoretically, including trapped ions [6], photon pairs [7], and atomic ensembles [8]. For these systems to work efficiently, maximally entangled states are required. However, such systems are extremely susceptible to the environment and this uncontrollable interaction may result in decoherence, thereby degrading the entanglement [9]. On the other hand, it is also possible that the interaction between quantum systems and the environment may lead to the formation of entangled states [10]. Thus, understanding the generation of entanglement under the influence of dissipative effects, such as temperature, has become one of the most important problems in the processing of quantum information [11]. Pioneering works as presented in [12] showed a generation of atomic entanglement by a single-mode thermal field. Galve et al. [13] showed the existence of entangled states even at high temperatures, considering a system of two coupled harmonic oscillators. In [14], the authors showed that entanglement can always arise in the interaction of an arbitrarily large system (thermal field) in any mixed state with a single qubit (two-level atom) in a pure state. The case of two atoms interacting with a single mode of a thermal field has been studied by [15].

Unlike conventional single-mode cavity QED architectures, the microtoroidal cavity containing two counter-propagating whispering gallery modes (WGMs) has attracted considerable attention 
due to the small volume and ultrahigh-quality factor $Q$ that minimize losses (decoherence) and allows a strong coupling between atomic systems and the electromagnetic field [16]. Additionally, the WGMs allow drastic reduction of energy for being useful for observing nonlinear effects [17]. In [18], the authors have shown that based on the effective dipole-dipole interaction between two nitrogen-vacancy centers mediated by the WGMs, it is possible to have quantum information transfer and entanglement through Raman transitions combined with laser fields. The generalization for the case of three NVCs interacting and coupled to a WGMs microresonator has been studied in [19]. The control of two-atom entanglement with two thermal fields by the hopping strength and the detuning between the atomic transition and the cavities has been demonstrated in [20]. In addition, the role of dipole-dipole interaction and scattering strength in the generation of entanglement between two and three two-level atoms coupled to the WGMs cavity has been investigated in [21]. However, despite previous work, the persistence of entanglement at high temperatures between two-level atoms coupled to the WGMs by adjusting the parameters has not been studied yet. In addition, an important factor that must be taken into account is the interaction between the WGMs due to experimental difficulties that demand a high precision manufacturing [22]. In fact, the high quality factor $Q$, necessary for strong interactions between atom and cavity, is related to the interaction between the WGMs [23]. Therefore, it would be interesting to investigate how the effects of the thermal field, at high temperatures, can be supplied through controllable parameters of the system for the maintenance of the entanglement, with high fidelity, between two two-level atoms coupled to a microtoroidal cavity.

Here, we propose to study the generation of entanglement and quantum state transfer in a system comprising a pair of two-level dipole-dipole interacting atoms coupled to a microtoroidal resonator. In this case, the two atoms are individually coupled with the two WGMs of a microtoroidal resonator through their evanescent fields. We compute the atom-atom entanglement using the negativity for different sets of parameters of the system. Two cases are investigated, the ideal and non-ideal cavity regimes, where the latter allows the direct coupling between the WGMs of the resonator. In the ideal cavity regime, we show that the entanglement is correlated to the dipole-dipole interaction and the average number of photons when the modes of the resonator are prepared in a thermal state even at high temperatures. For the non-ideal cavity regime, a counter-intuitive result is obtained. The imperfections also offer the advantage of generating maximally entangled states for a two-atom subsystem with maximum fidelity.

Our paper is organized as follows. First, in Section 2, we present the model and description of the physical system. Then, in Section 3, we discuss and show the results for the measurement of atom-atom entanglement and fidelity for different parameters. The conclusions are summarized in Section 4 .

\section{Theoretical Model}

We consider a system of two two-level atoms interacting through dipole-dipole interaction, where each of them is coupled simultaneously to a microtoroidal cavity as shown in Figure 1. We denote the atoms by label $i=1,2$ and assume that the two atoms are close enough so that the dipole-dipole interaction cannot be ignored. The cavity supports a pair of WGM's of frequency $\omega_{c}$ coupled to two atoms with transition frequency $\omega_{\text {eg }}^{i}$. 

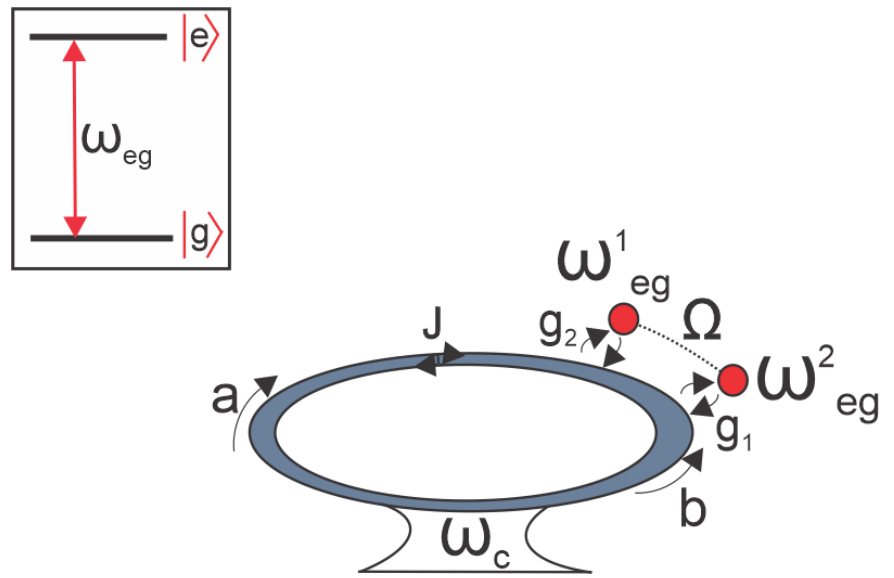

Figure 1. Schematic representation of the atoms-microtoroid system. The identical two-level atoms interacting via dipole-dipole interaction $\Omega$, where each of them is coupled simultaneously to the two whispering gallery modes (WGMs). The two-mode are coupled to each other via $J$ due to the cavity imperfections.

In addition, we consider that there is a direct coupling between the two WGMs due to backscattering induced by cavity imperfections. According to the above scheme, the Hamiltonian of the system can be written in the form [24-26]:

$$
\begin{aligned}
\hat{H}= & \sum_{i=1,2} \frac{1}{2} \hbar \omega_{e g}^{i} \sigma_{z}^{i}+\hbar \omega_{c}\left(\hat{a}^{\dagger} \hat{a}+\hat{b}^{\dagger} \hat{b}\right)+\hbar\left(g_{1}^{*} \hat{a}^{\dagger} \sigma_{1}^{-}+g_{1} \hat{a} \sigma_{1}^{\dagger}\right)+\hbar\left(g_{1} \hat{b}^{\dagger} \sigma_{1}^{-}+g_{1}^{*} \hat{b} \sigma_{1}^{\dagger}\right)+ \\
& \hbar\left(g_{2}^{*} \hat{a}^{\dagger} \sigma_{2}^{-}+g_{2} \hat{a} \sigma_{2}^{\dagger}\right)+\hbar\left(g_{2} \hat{b}^{\dagger} \sigma_{2}^{-}+g_{2}^{*} \hat{b} \sigma_{2}^{\dagger}\right)+\hbar \Omega\left(\sigma_{1}^{+} \sigma_{2}^{-}+\sigma_{1}^{-} \sigma_{2}^{+}\right)+\hbar J\left(\hat{a}^{\dagger} \hat{b}+\hat{a} \hat{b}^{\dagger}\right)
\end{aligned}
$$

where $\hat{a}^{\dagger}(\hat{a})$ and $\hat{b}^{\dagger}(\hat{b})$ are the creation (annihilation) operators of photons for the left and right modes of the resonator fields, respectively, $\sigma_{i}^{+}=|e\rangle_{i}\langle g|$ and $\sigma_{i}^{-}=|g\rangle_{i}\langle e|$ are the raising and lowering operators of the atom $i, g_{i}\left(g_{i}^{*}\right)$ is the coupling constant between the atom $i$ and the left (right) modes of the resonator field, $\Omega$ is the coupling constant of the dipole-dipole interaction between the atoms, and $J$ is the coupling constant of the interaction between the two WGMs. The first and second terms of the Hamiltonian Equation (1) represent the free energies of the atoms and resonator modes, respectively. The third and fourth (fifth and sixth) terms describe the coupling between the atom 1 (atom 2) with the two WGMs of the resonator. The last two terms take into account the atom-atom coupling via dipole-dipole interaction and the coupling between the two modes, respectively. For the sake of simplicity, we assume that the atomic transition frequency of each atom is in resonance with the cavity $\left(\omega_{e g}^{i}=\omega_{c}=\omega\right)$ and in the frame of the interaction representation with respect to $H_{0}=\sum_{i=1,2}(\hbar \omega / 2) \sigma_{z}^{i}+\hbar \omega\left(\hat{a}^{\dagger} \hat{a}+\hat{b}^{\dagger} \hat{b}\right)$, the interaction Hamiltonian, in rotating wave approximation (RWA) is given by [24-26]:

$$
\begin{aligned}
\hat{H}_{I}= & \hbar\left(g_{1}^{*} \hat{a}^{\dagger} \sigma_{1}^{-}+g_{1} \hat{a} \sigma_{1}^{\dagger}\right)+\hbar\left(g_{1} \hat{b}^{\dagger} \sigma_{1}^{-}+g_{1}^{*} \hat{b} \sigma_{1}^{\dagger}\right)+\hbar\left(g_{2}^{*} \hat{a}^{\dagger} \sigma_{2}^{-}+g_{2} \hat{a} \sigma_{2}^{\dagger}\right)+ \\
& \hbar\left(g_{2} \hat{b}^{\dagger} \sigma_{2}^{-}+g_{2}^{*} \hat{b} \sigma_{2}^{\dagger}\right)+\hbar \Omega\left(\sigma_{1}^{+} \sigma_{2}^{-}+\sigma_{1}^{-} \sigma_{2}^{+}\right)+\hbar J\left(\hat{a}^{\dagger} \hat{b}+\hat{a} \hat{b}^{\dagger}\right) .
\end{aligned}
$$

In this case, the time-dependent density operator for the atoms-microtoroid system follows a unitary time evolution generated by the evolution operator $U(t)=e^{-\frac{i H_{I}}{\hbar} t}$. For the sake of simplicity, we consider $g_{1}=g_{1}^{*}=g_{2}=g_{2}^{*}=|g|$ and $J=0$ (ideal resonator case). Using the Hamiltonian (2) and Taylor expansions of sine and cosine functions in the basis $\{|e e\rangle,|e g\rangle,|g e\rangle,|g g\rangle\}$, we obtain an analytical form of the evolution operator $U(t)$ given by:

$$
U(t)=\left(\begin{array}{llll}
U_{11} & U_{12} & U_{13} & U_{14} \\
U_{21} & U_{22} & U_{23} & U_{24} \\
U_{31} & U_{32} & U_{33} & U_{34} \\
U_{41} & U_{42} & U_{43} & U_{44}
\end{array}\right),
$$


where

$$
\begin{gathered}
U_{11}=1+2(\hat{a}+\hat{b}) \frac{w}{\lambda}\left(\hat{a}^{\dagger}+\hat{b}^{\dagger}\right), \quad U_{12}=U_{13}=(\hat{a}+\hat{b}) \frac{z}{\theta}, \quad U_{14}=2(\hat{a}+\hat{b}) \frac{w}{\lambda}(\hat{a}+\hat{b}), \\
U_{41}=2\left(\hat{a}^{\dagger}+\hat{b}^{\dagger}\right) \frac{w}{\lambda}\left(\hat{a}^{\dagger}+\hat{b}^{\dagger}\right), \quad U_{21}=U_{31}=\frac{z}{\theta}\left(\hat{a}^{\dagger}+\hat{b}^{\dagger}\right), \quad U_{24}=U_{34}=\frac{z}{\theta}(\hat{a}+\hat{b}), \\
U_{23}=U_{32}=\frac{e^{\frac{-i}{2}(\varepsilon+\theta) \tau}}{4 \theta}\left\{\left(1-e^{i \theta \tau}\right) \varepsilon-2 \theta e^{\frac{i}{2}(3 \varepsilon+\theta) \tau}+\theta\left(1+e^{i \theta \tau}\right)\right\}, \\
U_{42}=U_{43}=\left(\hat{a}^{\dagger}+\hat{b}^{\dagger}\right) \frac{z}{\theta}, \quad U_{44}=1+2\left(\hat{a}^{\dagger}+\hat{b}^{\dagger}\right) \frac{w}{\lambda}(\hat{a}+\hat{b}), \\
U_{22}=U_{33}=\frac{e^{\frac{-i}{2}(\varepsilon+\theta) \tau}}{4 \theta}\left\{\left(1-e^{i \theta \tau}\right) \varepsilon+2 \theta e^{\frac{i}{2}(3 \varepsilon+\theta) \tau}+\theta\left(1+e^{i \theta \tau}\right)\right\},
\end{gathered}
$$

with

$$
\tau=g t, \quad \varepsilon=\frac{\Omega}{g}, \quad \lambda=4\left(\hat{a}^{\dagger} \hat{a}+\hat{b}^{\dagger} \hat{b}+1\right), \quad \theta=\sqrt{16\left(\hat{a}^{\dagger} \hat{a}+\hat{b}^{+} \hat{b}+1\right)+\varepsilon^{2}},
$$

and

$$
w=e^{-i \frac{\varepsilon}{2} \tau}\left\{\cos \left(\frac{\theta}{2} \tau\right)+i \frac{\varepsilon}{\theta} \sin \left(\frac{\theta}{2} \tau\right)\right\}-1, \quad z=e^{\frac{-i}{2}(\varepsilon+\theta) \tau}\left(1-e^{i \theta \tau)} .\right.
$$

We assume that the resonator modes are initially prepared in a thermal state at temperature $T$ with the probability distribution $P(n)$. In this case, to determine the mean photon number in thermal equilibrium we have used the Boltzmann distribution. The thermal fields are driven by an external field, prepared in a mixture of fock states, completely described in terms of a mean photon number $\bar{n}_{i}$, which can be written as:

$$
\rho_{R}(0)=\rho_{R_{1}}(0) \rho_{R_{2}}(0)
$$

where

$$
\begin{gathered}
\rho_{R_{i}}(0)=\sum_{n_{i}} P_{i}\left(n_{i}\right)\left|n_{i}\right\rangle\left\langle n_{i}\right|, \\
P_{i}\left(n_{i}\right)=\frac{\bar{n}_{i}^{n_{i}}}{\left(1+\bar{n}_{i}\right)^{n_{i}+1}} \quad(i=1,2)
\end{gathered}
$$

with $\bar{n}_{i}=\left(e^{\frac{\hbar \omega}{k_{B} T}}-1\right)^{-1}$ being the mean photon number that relates to the environment temperature, where $k_{B}$ is the Boltzmann constant, $\omega$ is the frequency of the field of the resonator, and $T$ is the equilibrium temperature.

Thus, the density operator as a function of the time can be represented by:

$$
\rho(t)=e^{-i H_{I} t / \hbar}\left(\rho_{R_{i}}(0) \otimes \rho_{a}(0)\right) e^{i H_{I} t / \hbar} .
$$

To investigate the atom-atom entanglement, we need to obtain the time-dependent reduced atomic density operator, that is done by tracing over the resonator field variables:

$$
\begin{aligned}
\rho_{a}(t) & =\operatorname{Tr}_{R_{i}}[\rho(t)] \\
& =\sum_{n, m} P_{i}\left(n_{i}\right)\left\langle m\left|e^{-i H t / \hbar}\right| n\right\rangle \rho(t)\left\langle n\left|e^{i H t / \hbar}\right| m\right\rangle .
\end{aligned}
$$

In order, we use the criterion proposed by Vidal and Werner [27] to measure the degree of entanglement between the two atoms by calculating the negativity, defined in terms of the negative eigenvalues of the partial transposed of the reduced density matrix:

$$
\mathcal{N}=-2 \sum_{i} \mu_{i}^{-}
$$




\section{Entanglement Dynamics between the Two Atoms}

Our focus at this stage is to study the entanglement between two atoms analyzing the importance of the most relevant parameters of the model. To begin we assume that the atoms are initially prepared in a superposition state, $\cos \theta|e g\rangle+\sin \theta|g e\rangle$. Without loss of generality, we assume that $\bar{n}_{1}=\bar{n}_{2}=\bar{n}$. Then, we investigate the entanglement dynamics when the two atoms are coupled to an ideal microtoroidal cavity $(J=0)$. After, we generalize for the case of the possible deformation in the cavity $(J \neq 0)$ to investigate how the imperfection modifies or determines the dynamics of the entanglement as well as the fidelity.

\subsection{Two Atoms Coupled to an Ideal Resonator $(J=0)$}

Initially, we consider that one atom is in its ground state and the other in an excited state $|g e\rangle$ (i.e., for $\theta=\pi / 2$ ). The density operator of the atoms can be written as:

$$
\rho_{a}(t)=\left(\begin{array}{cccc}
A_{e e} & 0 & 0 & 0 \\
0 & B_{g e} & E_{e g}^{*} & 0 \\
0 & E_{g e} & C_{e g} & 0 \\
0 & 0 & 0 & D_{g g}
\end{array}\right)
$$

where the elements of the density matrix are:

$$
\begin{array}{r}
A_{e e}=\sum_{n} P_{n} n(n-1)\left|U_{1,2 n}\right|^{2} \\
B_{g e}=\sum_{n} P_{n}\left|U_{2,2 n}\right|^{2} \\
C_{e g}=\sum_{n} P_{n}\left|U_{2,3 n}\right|^{2} \\
D_{e e}=\sum_{n} P_{n} n(n+1)\left|U_{4,2 n}\right|^{2} \\
E_{g e}=E_{e g}^{*}=\sum_{n} P_{n} n(n+1) U_{2,2 n} U_{2,3 n}^{*} .
\end{array}
$$

Let us first consider that the field is initially prepared in its vacuum state $(\bar{n}=0)$ and the atoms are close enough so that the dipole-dipole interaction is allowed. Thus, from Equation (7) it was possible to calculate, numerically, the negativity when the atoms are initially prepared in a state $|g e\rangle$ for different values of the dipole strength as shown in Figure 2a. We can see that the atom-atom entanglement is strongly dependent on the dipole interaction. One can note that for $\Omega=0.1 \mathrm{~g}$ (line dotted) the entanglement has a monotonically increasing oscillatory behavior, reaching a value near $\sim 0.998$. It is also interesting to note that, even when there is no dipole-dipole interaction between the atoms, $\Omega=0$ (solid line), still an amount of entanglement survives at some specific time. We observed that, as the dipole interaction is a natural mechanism for generating entanglement between the atoms, even for $\Omega=0.001 \mathrm{~g}$ (line solid) there are small bumps indicating a persistence of the entanglement in certain time intervals.

The results shown in Figure 2a agree with the ones discussed for single-mode cavity QED [12,15]. But, there is a difference in the present case, which is indeed the enhancement of the entanglement peaks present in the microtoroidal system when compared with the single-mode cavity QED. In order, to analyze the influence of temperature for this system, i.e., the importance of the average number of photons $(\bar{n} \neq 0)$, we consider the entanglement for a fixed dipole strength $(\Omega=0.1 \mathrm{~g})$. The results are shown in Figure 2b. Note that when the temperature is increased the entanglement decreases, as expected. In fact, the exchange of energy between the atoms and the field increases with the average number of photons, increasing the decoherence. However, it is noted that, even for high temperatures (large number of $\bar{n}=100$ ) a certain amount of entanglement still survives (see inset in Figure $2 b$ ). 


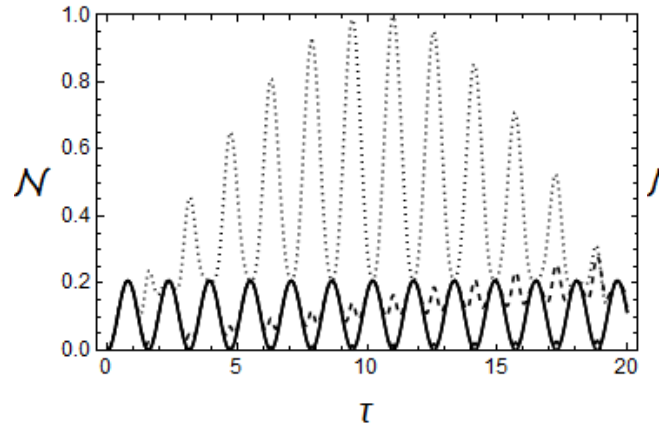

(a)

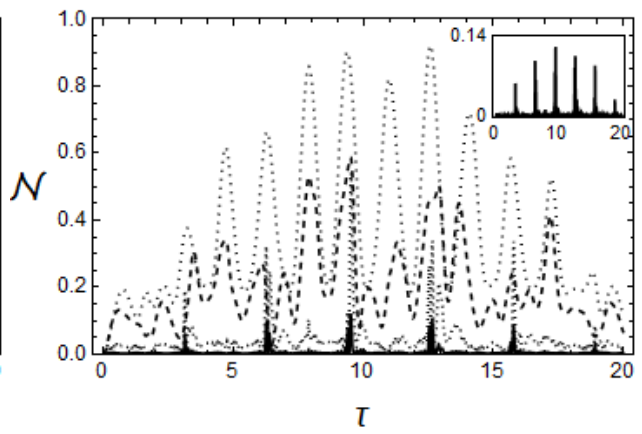

(b)

Figure 2. Negativity as function of the normalized time $\tau(\mathbf{a})$ for the resonator in the vacuum state $(\bar{n}=0)$ and dipole constant coupling $\Omega=0.1 \mathrm{~g}$ (dotted); $\Omega=0.01 \mathrm{~g}$ (dashed); $\Omega=0.001 \mathrm{~g}$ (solid); and $\Omega=0$ (solid); and (b) for the resonator in a thermal state and dipole constant coupling $\Omega=0.1 \mathrm{~g}$ and mean number photons $\bar{n}=0.1$ (dotted); $\bar{n}=1.0$ (dashed); $\bar{n}=10$ (solid); and $\bar{n}=100$ (solid). Inset: The zoom for $\bar{n}=100$.

\subsection{Two Atoms Coupled to a Non-Ideal Resonator $(J \neq 0)$}

In this section, we assume that there is a direct coupling between the two WGMs, i.e., $J \neq 0$. In this case, the two atoms are initially prepared in different states. In Figure 3, we show the negativity between the two atoms as a function of the normalized time and of the dipole coupling for two different initial preparations of atomic states: In (a) for the state $|g e\rangle$ and (b) for $(|g e\rangle+|e g\rangle) / \sqrt{2}$. The result shows the importance of the coupling between the modes on the dynamical evolution of the atomic entanglement for a fixed dipole strength $\Omega=0.1 \mathrm{~g}$. As can be seen, there is a great influence of the initial state on the degree of entanglement of the atoms.

In Figure 3a, we can observe that for small values of $\mathrm{J} / \mathrm{g}$ we have only entanglement isles so that all the dynamics of the entanglement is linked directly with the imperfections in the resonator. However, this behavior, as shown the Figure $3 b$, is completely different if the atoms are initially prepared in a maximally entangled state. We observed that imperfection brings advantages for the generation of maximum entanglement between the atoms reaching stability for large values of $\mathrm{J} / \mathrm{g}$.

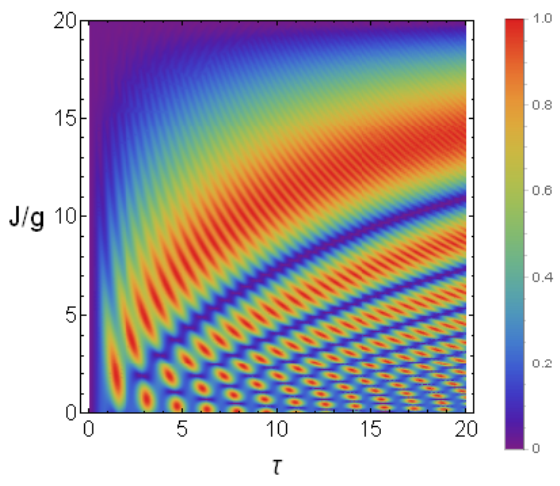

(a)

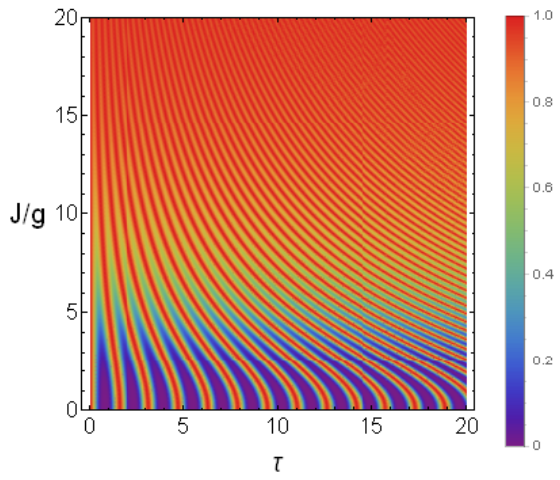

(b)

Figure 3. Negativity as function of the normalized time $\tau$ and of the interaction between the two counter-propagating modes for the mean number photons $\bar{n}=0.01$ and the atoms prepared in initial state (a) $|g e\rangle ;(\mathbf{b})(|g e\rangle+|e g\rangle) / \sqrt{2}$.

Next, it is possible to estimate the performance of our scheme, using the fidelity as defined by [28]. Experimentally, it is difficult to control precisely the interaction between the modes described through imperfection, since it depends on the manufacturing processes. Consequently, it is important 
to determine how imperfections can modify or determine the fidelity. Figure 4 shows the fidelity for the situation when the two atoms are prepared in the superposition state, $\cos \theta|g e\rangle+\sin \theta|e g\rangle$, and with $\Omega=0.1 \mathrm{~g}$. Figure 4a shows that the transfer of the prepared state is completely reached when $\theta=\pi / 4$ for $J=0.1 \mathrm{~g}$, showing a high fidelity in the transference of the maximally entangled state between the atoms, while for $\theta=\pi / 6$ the maximum fidelity possible is approximately $75 \%$. Figure $4 \mathrm{~b}$ shows that the presence of deformation can decrease the time of entangled state transference. The robustness of the fidelity in the regime of deformations can be useful in practical applications.

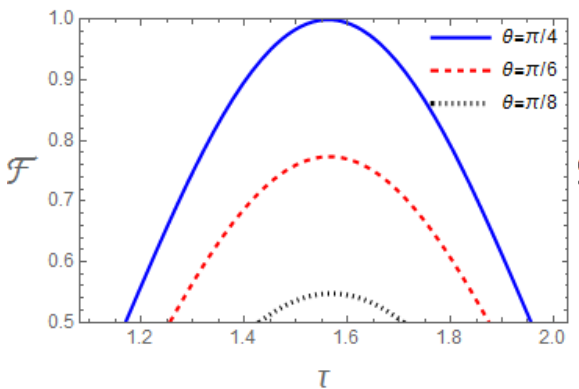

(a)

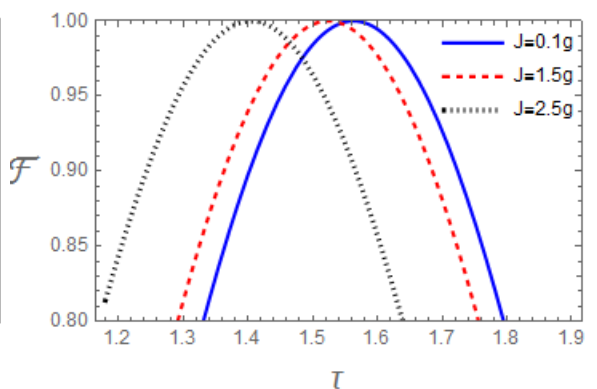

(b)

Figure 4. Fidelity as function of the normalized time $\tau$ with mean number photons $\bar{n}=0.01$ and (a) for interaction between the modes $J=0.1 \mathrm{~g}$ and different $\theta$ and (b) for $\theta=\pi / 4$ and different $J$.

\section{Conclusions}

In summary, we have explored the entanglement dynamics between two identical two-level atoms interacting via dipole-dipole interaction and coupled simultaneously to the two WGMs microtoroidal resonator in the presence of a thermal field. We have shown that the entanglement is strongly dependent on the dipole coupling strength and the mean photon number of the thermal field. In this latter case, we show that, differently from the ideal case $(\bar{n}=0)$, for certain parameters of the system, the atomic entanglement still survives even at high temperatures, in contrast to schemes based on single-mode cavity QED. It is also noted that, even when there is structural deformation in the microtoroidal resonator $(J \neq 0)$, it is possible to generate maximum atom-atom entanglement states. In addition, the transfer of a superposition state between the two atoms via the WGMs is reached with high fidelity. This also occurs in both ideal and non-ideal regimes, that is a favorable point this kind of system, given that, in the real world, one has to face non zero temperature regimes as well as losses. Therefore, the present study is valuable for understanding the relationship between dissipative effects, such as thermal fields, and unveil a set of system's parameters that may be of great importance for applications in quantum information processing tasks.

Author Contributions: Conceptualization, E.H.S.S. and J.A.R.; methodology, E.H.S.S. and J.A.R.; software, E.H.S.S.; validation, E.H.S.S. and J.A.R.; formal analysis, E.H.S.S. and J.A.R.; investigation, E.H.S.S. and J.A.R.; resources, J.A.R.; data curation, E.H.S.S. and J.A.R.; writing-original draft preparation, E.H.S.S.; writing-review and editing, E.H.S.S. and J.A.R.; visualization, E.H.S.S.; supervision, J.A.R.; project administration, J.A.R.; funding acquisition, J.A.R. All authors have read and agreed to the published version of the manuscript.

Funding: This research was funded by Conselho Nacional de Desenvolvimento Científico e Tecnológico (CNPq) grant number 140039/2016-3 (http:/ / www.carloschargas.cnpq.br), Coordenação de Aperfeiçoamento de Pessoal de Nível Superior (CAPES) grant number 1247693/2013 (https:/ /www.capes.gov.br), Fundação de Apoio a Pesquisa do Estado de São Paulo (FAPESP) (http://www.fapesp.br), and Instituto Nacional de Ciência e Tecnologia (INCT) (http:/ / www.inct-iq@if.ufrj.br).

Conflicts of Interest: The authors declare no conflict of interest. 


\section{References}

1. Gisin, N.; Ribordy, G.; Tittel, W.; Zbinden, H. Quantum cryptography. Rev. Mod. Phys. 2002, 74, 145-195. [CrossRef]

2. Arute, F.; Arya, K.; Babbush, R.; Bacon, D.; Bardin, J.C.; Barends, R.; Biswas, R.; Boixo, S.; Brandao, F.G.; Buell, D.A.; et al. Quantum supremacy using a programmable superconducting processor. Nature 2019, 574, 505-510. [CrossRef]

3. Bennett, C.H.; Brassard, G.; Crépeau, C.; Jozsa, R.; Peres, A.; Wootters, W.K. Teleporting an unknown quantum state via dual classical and Einstein-Podolsky-Rosen channels. Phys. Rev. Lett. 1993, 70, 1895-1899. [CrossRef] [PubMed]

4. Walther, H.; Varcoe, B.T.H.; Englert, B.G.; Becker, T. Cavity quantum electrodynamics. Rep. Prog. Phys. 2006, 69, 1325-1382. [CrossRef]

5. Kimble, H.J. The quantum internet. Nature 2008, 453, 1023-1030. [CrossRef]

6. Leibfried, D.; Blatt, R.; Monroe, C.; Wineland, D. Quantum dynamics of single trapped ions. Rev. Mod. Phys. 2003, 75, 281-324. [CrossRef]

7. Thompson, J.K.; Simon, J.; Loh, H.; Vuletić, V. A high-brightness source of narrowband, identical-photon pairs. Science 2006, 313, 74-77. [CrossRef] [PubMed]

8. Duan, L.M.; Lukin, M.D.; Cirac, J.I.; Zoller, P. Long-distance quantum communication with atomic ensembles and linear optics. Nature 2001, 414, 413-418. [CrossRef] [PubMed]

9. Plenio, M.B.; Knight, P.L. The quantum-jump approach to dissipative dynamics in quantum optics. Rev. Mod. Phys. 1998, 70, 101-144. [CrossRef]

10. Plenio, M.B.; Huelga, S.F.; Beige, A.; Knight, P.L. Cavity-loss-induced generation of entangled atoms. Phys. Rev. A 1999, 59, 2468-2475. [CrossRef]

11. Lin, Q.; He, B.; Xiao, M. Entangling Two Macroscopic Mechanical Resonators at High Temperature. Phys. Rev. Appl. 2020, 13, 034030. [CrossRef]

12. Kim, M.S.; Lee, J.; Ahn, D.; Knight, P.L. Entanglement induced by a single-mode heat environment. Phys. Rev. A 2002, 65, 040101. [CrossRef]

13. Galve, F.; Pachón, L.A.; Zueco, D. Bringing Entanglement to the High Temperature Limit. Phys. Rev. Lett. 2010, 105, 180501. [CrossRef] [PubMed]

14. Bose, S.; Fuentes-Guridi, I.; Knight, P.L.; Vedral, V. Subsystem Purity as an Enforcer of Entanglement. Phys. Rev. Lett. 2001, 87, 050401. [CrossRef] [PubMed]

15. Aguiar, L.S.; Munhoz, P.P.; Vidiella-Barranco, A.; Roversi, J.A. The entanglement of two dipole-dipole coupled atoms in a cavity interacting with a thermal field. J. Opt. B Quantum Semiclass. Opt. 2005, 7, S769-S771. [CrossRef]

16. Spillane, S.M.; Kippenberg, T.J.; Painter, O.J.; Vahala, K.J. Ideality in a Fiber-Taper-Coupled Microresonator System for Application to Cavity Quantum Electrodynamics. Phys. Rev. Lett. 2003, 91, 043902. [CrossRef]

17. Spillane, S.; Kippenberg, T.; Vahala, K. Ultralow-threshold Raman laser using a spherical dielectric microcavity. Nature 2002, 415, 621-623. [CrossRef]

18. Li, P.B.; Gao, S.Y; Li, F.L. Quantum-information transfer with nitrogen-vacancy centers coupled to a whispering-gallery microresonator. Phys. Rev. A 2011, 83, 054306. [CrossRef]

19. Song, W.; Yang, W.; Chen, Q.; Hou, Q.; Feng, M. Entanglement dynamics for three nitrogen-vacancy centers coupled to a whispering-gallery-mode microcavity. Opt. Express 2015, 23, 13734-13751. [CrossRef]

20. Shen, L.T.; Yang, Z.B.; Wu, H.Z.; Chen, X.Y.; Zheng, S.B. Control of two-atom entanglement with two thermal fields in coupled cavities. J. Opt. Soc. Am. B 2012, 29, 2379-2385. [CrossRef]

21. Sousa, E.H.; Roversi, J. The role of dipole-dipole interaction and the scattering strength in entanglement dynamics between atoms surrounding a microtoroidal cavity. Eur. Phys. J. Plus 2019, 134, 607. [CrossRef]

22. Rahachou, A.I.; Zozoulenko, I.V. Effects of boundary roughness on a $Q$ factor of whispering-gallery-mode lasing microdisk cavities. J. Appl. Phys. 2003, 94, 7929-7931. [CrossRef]

23. Kippenberg, T.J.; Spillane, S.M.; Vahala, K.J. Modal coupling in traveling-wave resonators. Opt. Lett. 2002, 27, 1669-1671. [CrossRef] [PubMed]

24. Dayan, B.; Parkins, A.; Aoki, T.; Ostby, E.; Vahala, K.; Kimble, H. A photon turnstile dynamically regulated by one atom. Science 2008, 319, 1062-1065. [CrossRef] 
25. Aoki, T.; Parkins, A.S.; Alton, D.J.; Regal, C.A.; Dayan, B.; Ostby, E.; Vahala, K.J.; Kimble, H.J. Efficient Routing of Single Photons by One Atom and a Microtoroidal Cavity. Phys. Rev. Lett. 2009, 102, 083601. [CrossRef]

26. Sousa, E.H.S.; Roversi, J.A. Selective Engineering for Preparing Entangled Steady States in Cavity QED Setup. Quantum Rep. 2019, 1, 63-70. [CrossRef]

27. Vidal, G.; Werner, R.F. Computable measure of entanglement. Phys. Rev. A 2002, 65, 032314. [CrossRef]

28. Jozsa, R. Fidelity for Mixed Quantum States. J. Mod. Opt. 1994, 41, 2315-2323. [CrossRef]

(C) 2020 by the authors. Licensee MDPI, Basel, Switzerland. This article is an open access article distributed under the terms and conditions of the Creative Commons Attribution (CC BY) license (http:/ / creativecommons.org/licenses/by/4.0/). 